\title{
Letrozole Therapy for Obstructive Azoospermic Men before in vitro Fertilization (IVF) treatment with Percutaneous Epididymal Sperm Aspiration
}

\author{
Mauro Bibancos ${ }^{1}$, Melissa Cavagnoli ${ }^{1}$, Tatiana C. S. Bonetti ${ }^{2}$, Erika Semaco ${ }^{1}$, Eduardo L. A. Motta ${ }^{1,2}$, Paulo C. \\ Serafini ${ }^{1,2}$ \\ ${ }^{1}$ Huntington - Reproductive Medicine - Sao Paulo - Brazil \\ 2Department of Gynecology, Paulista School of Medicine, Federal University of São Paulo (EPM-UNIFESP)
}

\begin{abstract}
Objective: The aim of this study was to report our preliminary experience regarding the use of letrozole in men with obstructive azoospermia (OA) undergoing percutaneous epididymal sperm aspiration (PESA) for in vitro fertilization treatment using intracytoplasmic sperm injection (ICSI), who had a very low sperm recovery upon PESA and unsuccessful ICSI. Our hypothesis was that letrozole therapy could improve testicular function by increasing serum gonadotropins and T levels, stimulate testicle germ cells and, most importantly, that it enhanced the motile sperm count at a second attempt.
\end{abstract}

Methods: We report on our preliminary experience with letrozole therapy in 11 men with OA, who failed to achieve pregnancy in the first PESA-ICSI and did not have spermatozoa cryopreserved for a second attempt. The patients received 3 months of letrozole at $2.5 \mathrm{mg} /$ day and underwent PESA-ICSI after $6.1 \pm 3.8$ months. The patients were $48.6 \pm 9.6$ years old, and underwent at least two PESA procedures. We evaluated the total motile sperm count per PESA samples, as the increases in serum FSH, LH, and T levels after treatment.

Results: All parameters increased significantly at 3 months following letrozole therapy for most patients. The total motile sperm count increased from 100 to $500 \%$ compared to the first PESA.

Conclusion: Letrozole can be considered a reliable treatment to improve sperm recovery for men with OA undergoing PESA-ICSI cycles by increasing serum gonadotropins and testosterone $(\mathrm{T})$ levels, and-most importantly-the motile sperm count.

Keywords: Obstructive azoospermia, Letrozole, Percutaneous epididymal sperm aspiration, Intracytoplasmic sperm injection

\section{INTRODUCTION}

Azoospermia is defined by the absence of spermatozoa in the ejaculate after two assessments using centrifuged semen analysis. Its prevalence is about $1 \%$ in the general population, and $10-15 \%$ in infertile couples. It is classified as non-obstructive azoospermia (NOA) when clinical evidence of spermatogenesis is absent, and obstructive azoospermia (OA) which results from a seminal tract obstruction, while spermatogenesis is preserved (Donoso et al., 2007). Men with OA usually undergo percutaneous epididymis sperm aspiration (PESA) to recover spermatozoa directly from the epididymis in conjunction with intracytoplasmic sperm injection (ICSI), providing excellent treatment outcomes for those who wish to have children (Meng et al., 2005; Hsieh et al., 2007).

However, there is a decrease in sperm production in men with $O A$, suggested to be a testicular dysfunction resulted of endocrine disruption. The PESA procedure success is usually evaluated by the amount of motile sper- matozoa recovered (Van Perperstraten et al., 2001), and spare spermatozoa can be frozen after ICSI and thawed for a second treatment (Kovac et al., 2014). On the other hand, it is not always possible to recover sperm for cryopreservation, and increased sperm production and motility could contribute to more favorable PESA outcomes, and most likely better ICSI outcomes.

Empirical medical therapy using estrogen receptor modulators such as clomiphene citrate or tamoxifen citrate have been used to improve spermatogenesis, primarily focusing on the enhancement of intratesticular testosterone ( $T$ ) levels and stimulation of follicle-stimulating hormone (FSH) production (Schlegel, 2012). Recombinant FSH may also represent another alternative for infertile men in an attempt to increase sperm production (Foresta et al., 2009). Nevertheless, the response to FSH treatment depends on hormone receptor polymorphisms, and involves treatment with costly pharmacogenomics product (Selice et al., 2011). Another approach is the use of aromatase inhibitors, which have the ability to increase endogenous $T$ production without the associated increase in circulating estrogens seen with estrogen receptor modulators (Pavlovich et al., 2001).

Active spermatogenesis in men with hypospermatogenesis after letrozole trials (Femara; Sanofi-Novartis, Paris, France) have been reported (Patry et al., 2009; Zhao et al., 2014). Letrozole has also been used to treat men with non-obstructive azoospermia for 3 months, who then showed live spermatozoa in their ejaculate (Cavallini et al., 2013). It has been demonstrated that letrozole normalizes serum hormone levels and semen parameters in isolated hypogonadotropic hypogonadic obese men (Loves et al., 2008), and in those with severe oligospermia, low T levels, but normal gonadotropin levels (Gregoriou et al., 2012). Given this background, the aim of this study is to report our preliminary experience regarding the use of letrozole in men with OA undergoing PESA for ICSI, who had a very low sperm recovery at PESA and unsuccessful ICSI. Our hypothesis was that letrozole could improve testicular function by increasing serum gonadotropins and T levels, stimulate testicle germ cells and most importantly that it enhanced the motile sperm count at a second PESA.

\section{MATERIALS AND METHODS}

Eleven OA men undergoing PESA-ICSI treatment at Huntington - Reproductive Medicine, a private center in Brazil, during 2012 and 2013 fulfilled the criteria to receive letrozole therapy. The patients were diagnosed as presenting OA based on a clinical history, physical examination and azoospermia defined as the absence of sperm in the pellets of two centrifuged semen samples. The patients did not have indication for testicular biopsy because the $O A$ etiologies were vas deferens agenesis and vasectomy. The patients underwent PESA for sperm recovery for the first PESA-ICSI treatment. Eleven patients were eligible 


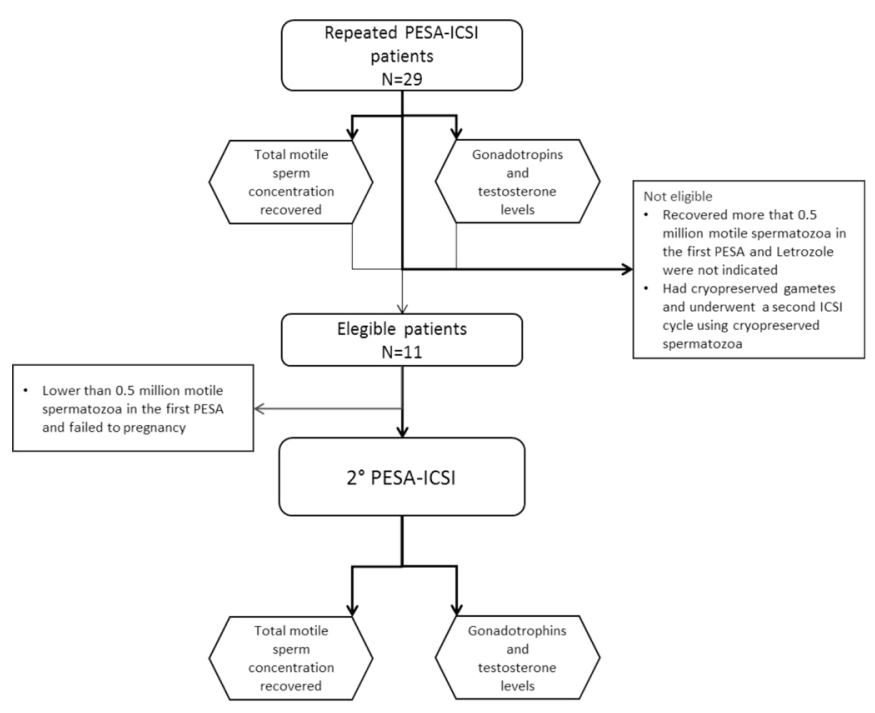

Figure 1: Flow diagram representing the study design

to receive letrozole therapy, as they had lower than 0.2 million motile spermatozoa in the first PESA, did not have sperm cryopreserved, failed to achieve pregnancy in the first PESA-ICSI cycle and were indicated to a second PESA-ICSI treatment. The patients had normal serum FSH $(<7.6 \mathrm{IU} / \mathrm{L})$ and $\mathrm{T}(>300 \mathrm{ng} / \mathrm{dL})$ (Figure 1). Institutional Review Board approval was not required as this study consisted of an off-label use of letrozole for OA men, which is an approved and registered medication routinely used. The off-label administration is authorized in Brazil under medical responsibility and no special authorization is required (ANVISA, 2005). Written informed consent was obtained from all patients for data use in scientific publication.

A clinical history and physical examination, measures of blood levels of FSH, luteinizing hormone (LH), T, and karyotyping were performed for each patient. The etiologies of OA were vas deferens agenesis $(n=3)$ and vasectomy $(n=8)$. The men received letrozole therapy for
3 months, at $2.5 \mathrm{mg}$ per day. These patients underwent another PESA-ICSI treatment after $6.1 \pm 3.8$ months. All the PESA procedures were carried out by the same surgeon, with the men under intravenous sedation with propofol (Diprivan; AstraZeneca, London, UK) and alfentanil (Rapifen; Janssen-Cilag Pty Ltd.). A 1-mL syringe with a $13 \times 4.5$ needle was inserted into the epididymis, and a negative pressure was created by drawing back the plunger. We carried out 8 to 10 aspirations in all areas of epididymis, head, body and tail, and then the needle was removed and the aspirate was flushed into a sterile microtube containing culture medium: HEPES-buffered Human Tubal Fluid (Irvine Scientific, Santa Ana, CA, USA) supplemented with 15\% Synthetic Serum Substitute (Irvine Scientific). An aliquot of this fluid was examined under the microscope to ascertain the presence of live motile spermatozoa. The aspirated material was washed with a minimal volume of culture medium. Samples were resuspended in $0.2 \mathrm{~mL}$ of the same culture medium and motile sperm counts were carried out in a Makler counting chamber (Irvine Scientific). A peripheral blood sample was obtained at the first and second PESA attempts for the measurement of serum hormone levels.

The total motile sperm count per PESA sample was calculated as the primary endpoint with respect to the efficacy of letrozole on increasing total motile sperm concentration from the first to second PESA procedures. Secondary endpoints included serum $\mathrm{FSH}, \mathrm{LH}$, and T levels after letrozole administration, and the correlations with motile sperm count. The data was collected and described per patient and presented as mean \pm standard deviation (SD); analyzed using STATA (StataCorp, Texas, USA), and non-parametric tests were applied because of the small number of patients included. The differences between the levels before and after therapy were assessed using the Wilcoxon signed rank test, and Spearman's rank analysis was carried out to evaluate any secondary correlations between endpoints; $P<0.05$ was considered statistically significant.

\section{RESULTS}

The individuals included in this study, aged $48.6 \pm 9.6$

\begin{tabular}{|l|l|c|c|c|c|c|c|c|c|}
\hline \multicolumn{2}{|l|}{ Table 1: Patient characteristics before and after letrozole treatment } \\
\hline$\#$ & Diagnosis & Age & $\begin{array}{c}\text { No. of } \\
\text { previous } \\
\text { PESA }\end{array}$ & $\begin{array}{c}\text { FSH } \\
(\mathrm{IU} / \mathrm{L}) \\
\text { pre } \\
\text { letrozole }\end{array}$ & $\begin{array}{c}\mathrm{FSH} \\
(\mathrm{IU} / \mathrm{L}) \\
\text { post } \\
\text { letrozole }\end{array}$ & $\begin{array}{c}\text { LH } \\
(\mathrm{IU} / \mathrm{L}) \\
\text { pre } \\
\text { letrozole }\end{array}$ & $\begin{array}{c}\text { LH } \\
(\mathrm{IU} / \mathrm{L}) \\
\text { post } \\
\text { letrozole }\end{array}$ & $\begin{array}{c}\text { Testosterone } \\
(\mathrm{ng} / \mathrm{ml}) \\
\text { pre } \\
\text { letrozole }\end{array}$ & $\begin{array}{c}\text { Testosterone } \\
(\mathrm{ng} / \mathrm{ml}) \\
\text { post } \\
\text { letrozole }\end{array}$ \\
\hline 1 & $\begin{array}{l}\text { deferens } \\
\text { agenesis }\end{array}$ & 42 & 2 & 7.6 & 18.1 & 3.4 & 13.1 & 371 & 871.0 \\
\hline 2 & $\begin{array}{l}\text { deferens } \\
\text { agenesis }\end{array}$ & 40 & 2 & 2.4 & 17.1 & 1.8 & 10.7 & 200 & 651.0 \\
\hline 3 & $\begin{array}{l}\text { deferens } \\
\text { agenesis }\end{array}$ & 32 & 2 & 2.6 & 6.8 & 2.8 & 10.3 & 323 & 1084.0 \\
\hline 4 & vasectomy & 55 & 3 & 3.0 & 13.8 & 2.4 & 11.7 & 435 & 681.0 \\
\hline 5 & vasectomy & 65 & 2 & 4.7 & 5.9 & 4.2 & 6.5 & 374 & 855.0 \\
\hline 6 & vasectomy & 43 & 2 & 4.5 & 12.7 & 6.3 & 35.7 & 951 & 942.0 \\
\hline 7 & vasectomy & 46 & 4 & 2.3 & 2.7 & 1.5 & 2.5 & 312 & 349.6 \\
\hline 8 & vasectomy & 53 & 3 & 5.4 & 11.2 & 4.3 & 5.5 & 305 & 916.0 \\
\hline 9 & vasectomy & 50 & 2 & 3.6 & 15.1 & 3.7 & 9.1 & 456 & 547.0 \\
\hline 10 & vasectomy & 55 & 3 & 5.3 & 12.1 & 3.7 & 8.7 & 487 & 678.0 \\
\hline 11 & vasectomy & 54 & 4 & 4.8 & 5.6 & 2.5 & 4.0 & 431 & 288.3 \\
\hline & Mean \pm SD & $48.6 \pm 9.6$ & $2.6 \pm 0.8$ & $4.2 \pm 1.7$ & $11.0 \pm 5.1$ & $3.2 \pm 1.4$ & $8.2 \pm 3.5$ & $422.3 \pm 193.7$ & $714.8 \pm 249.5$ \\
\hline
\end{tabular}




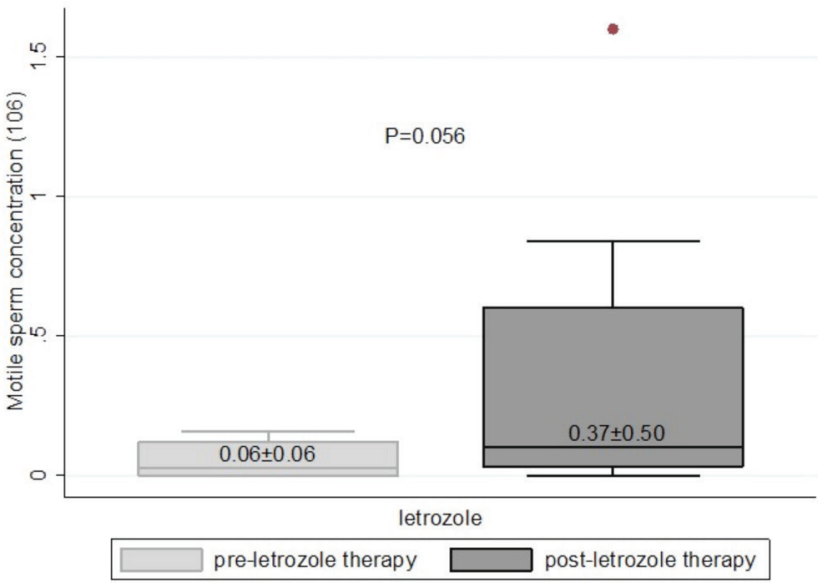

Figure 2: Motile sperm concentration before and after a 3-month administration of letrozole in men with obstructive azoospermia undergoing PESAICSI cycles.

years, presented a mean of $4.2 \pm 1.6 \mathrm{IU} / \mathrm{L}$ of $\mathrm{FSH}, 3.2 \pm 1.4$ $\mathrm{IU} / \mathrm{L}$ of $\mathrm{LH}, 422.3 \pm 193.7 \mathrm{ng} / \mathrm{ml}$ of $\mathrm{T}$, and underwent at least one or two PESA before and did not achieve pregnancy in the PESA-ICSI cycle. Patients received 3 months of letrozole therapy and underwent a subsequent PESA after treatment and all parameters were measured (Table 1). All hormone measurements and motile sperm concentration increased significantly in all patients. Table 2, Figures 2 and 3 summarize the results of motile sperm concentrations and serum $\mathrm{FSH}, \mathrm{LH}$, and T levels before and after 3 months of letrozole administration.

Three patients did not show any improvement in motile sperm concentration (patients 5, 9 and 11). Serum gonadotropin levels showed improvements in all patients. Concerning serum $T$ levels, one patient who already had high levels of basal testosterone (patient 6 ) did not show greater levels after therapy, despite higher FSH and LH levels and higher total motile sperm concentration.

The Spearman rank analysis showed no correlations between hormone levels and motile sperm concentrations upon pre- and post-letrozole PESA. The time between PESA procedures was also not associated with final motile sperm concentration. However, the age of the patients was inversely correlated with the motile sperm concentration after letrozole therapy $(r=-0.755, P=0.007)$.

We calculated the percentage of improvement in the total motile sperm count, which varied from $100 \%$ to higher than $500 \%$. This increase in the total motile sperm count showed a marginally significant correlation with serum $T$ levels $(r=0.524 ; P=0.098)$. In spite of the small number of patients, we calculated the sample power using a paired test comparing two related means, based on pre and post-motile sperm count. For this data the sample power was 0.46 (total motile sperm count - pre-treatment of 0.06 , -post-treatment of 0.4 , standard deviation of the difference equal to 0.5 ). Using the same parameters, the ideal sample size would have been 23 patients.

\section{DISCUSSION}

This study shows the preliminary data about the use of letrozole for men with OA undergoing PESA-ICSI cycles. Three months of therapy increased the motile sperm count in nine of 11 patients studied. Serum hormone levels also showed significant increases. It seems that the efficacy of letrozole was linked to patient age. The endocrine effects of FSH and the paracrine effects of $\mathrm{T}$ on the Sertoli cells primarily control spermatogenesis. However, because of the small number of patients evaluated, there was no statistically significant correlation between increases in motile sperm count and serum hormone levels in this study.

A number of mechanisms are supposed to affect spermatogenesis in men with $\mathrm{OA}$. The obstruction could result in distension of the reproductive tract, causing spermatogenic damage and reduced sperm production because of the lack of space for the spermatozoa produced (Peng et al., 2011). Interstitial fibrosis might affect the paracrine functions of the seminiferous tubules after vasectomy, leading to a reduction in spermatogenesis (Shiraishi et al., 2003). Spermatogenesis could also be impaired by cellular disturbances caused by electrolyte and transmembrane gradient imbalances. It is known that reactive oxygen species (ROS) are produced during spermatogenesis (Aitken \& Sawyer, 2003). However, they must be tightly regulated to prevent sperm damage (Aitken \& Krausz, 2001). A build up of spermatozoa in the epididymis following vasectomy might lead to a deregulation of ROS generation, causing oxidative stress. An additional and relevant mechanism for spermatogenic impairment could be the elevated rate of apoptosis. Apoptosis plays an important role in the normal testis (Rodriguez et al., 1997), eliminating $50-70 \%$ of germ cells at different stages of spermatogenesis prior to spermiogenesis (Garcia-Quevedo et al., 2014).

For OA patients, PESA is a safe and effective method of sperm retrieval, as it is a minimally invasive, simple procedure with few complications, and it is fast to perform. However, there is no agreement about which motile sperm minimum concentration is ideal. Usually, sperm and oocyte retrieval are performed on the same day with a successful ICSI fertilization rate in the range of $80-100 \%$ (Dohle et al., 1998). The pregnancy success rate of ICSI cycles depends on many other factors and runs around 30\% (Sunderam et al., 2013), which means a number of couples need to try a second treatment to achieve pregnancy.

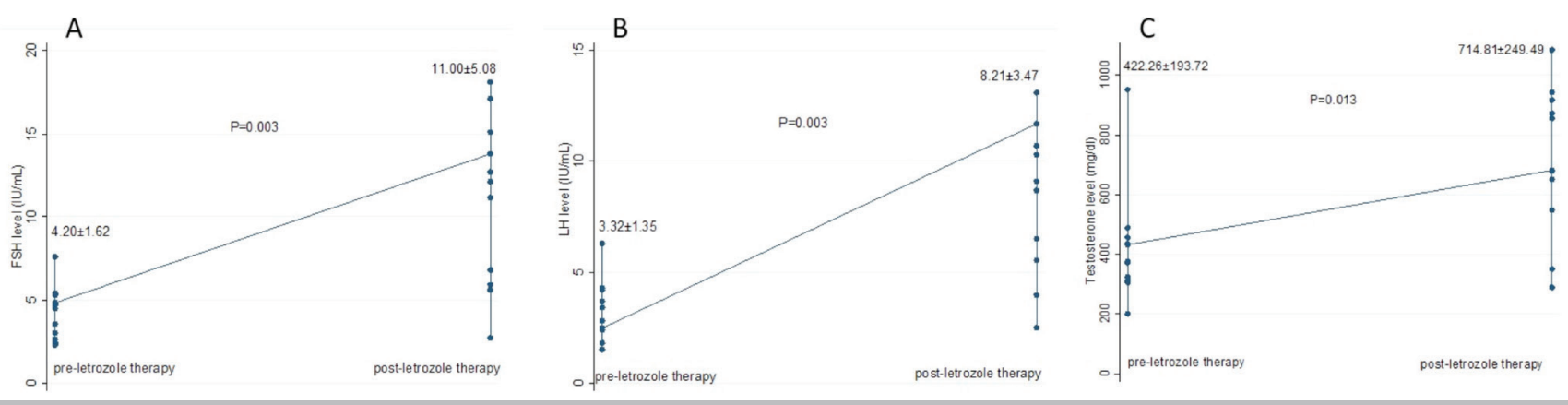

Figure 3: Serum FSH (A), LH (B), and T (C) levels before and after a 3-month administration of letrozole in men with obstructive azoospermia undergoing PESA-ICSI cycles. The data is presented as the mean \pm standard deviation. *Mann-Whitney nonparametric $U$ test. 
Table 2: Results on motile sperm concentration in the PESA before and after letrozole treatment

\begin{tabular}{|c|c|c|c|c|}
\hline$\#$ & $\begin{array}{c}\text { Motile sperm } \\
\text { concentration } \\
\text { pre letrozole }\left(\times 10^{6}\right)\end{array}$ & $\begin{array}{c}\text { Motile sperm } \\
\text { concentration } \\
\text { post letrozole }\left(\times 10^{6}\right)\end{array}$ & $\begin{array}{c}\text { Fold of increase } \\
\text { in motile sperm } \\
\text { concentration }\end{array}$ & $\begin{array}{c}\text { Percentage of increase in } \\
\text { motile sperm concentration }\end{array}$ \\
\hline 1 & 0.12 & 0.6 & 5.0 & $400 \%$ \\
\hline 2 & 0.06 & 0.22 & 3.7 & $267 \%$ \\
\hline 3 & 0.16 & 0.84 & 5.3 & $425 \%$ \\
\hline 4 & 0.03 & 0.1 & 3.3 & $233 \%$ \\
\hline 5 & 0.0001 & 0.00004 & 16000.0 & $-60 \%$ \\
\hline 6 & 0.0001 & 1.6 & 6000.0 & $>500 \%$ \\
\hline 7 & 0.0001 & 0.6 & 2.0 & $>500 \%$ \\
\hline 8 & 0.03 & 0.06 & 0.3 & $100 \%$ \\
\hline 9 & 0.12 & 0.03 & 3.0 & $-75 \%$ \\
\hline 10 & 0.02 & 0.06 & 0.03 & $200 \%$ \\
\hline 11 & 0.06 & 0.002 & & $-97 \%$ \\
\hline MeantSD & $0.06 \pm 0.06$ & $0.4 \pm 0.5$ & & \\
\hline
\end{tabular}

Letrozole therapy increases motile sperm counts by increasing serum gonadotropin and $\mathrm{T}$ levels in different spermatogenic conditions (Raman \& Schlegel, 2002; Loves et al., 2008; Patry et al., 2009; Cavallini et al., 2011; Saylam et al., 2011; Gregoriou et al., 2012; Cavallini et al., 2013; Zhao et al., 2014), which corroborates findings in our study.

Aromatase is present in testicular Leydig cells and aromatase inhibitors interact with this enzyme. Aromatase inhibitors act to limit estrogen production and maintain $T$ levels and could have efficacy in a variety of clinical settings, including impaired sperm production (Brodie, 1985). Clinically, the use of aromatase inhibitors has been applied to men with idiopathic infertility, with the intent of reducing estrogen effects on the male reproductive system, especially by reducing feedback inhibition via the hypothalamus-pituitary-gonadal axis. Inhibition of estrogen production by an aromatase inhibitor could be a potent stimulant for increased $\mathrm{FSH}$ and $\mathrm{LH}$ production and hence increase intra-testicular and circulating T levels (Santen, 1981).

However, individual differences might affect the efficacy of letrozole therapy. Aromatase from adipose tissue could increase aromatase activity, although there is no consensus in the literature (Schlegel, 2012). In addition, aromatase polymorphisms have been shown to affect various estrogen-dependent diseases (Hammoud et al., 2010), and could influence the efficacy of letrozole. In our patients, under off-label use of letrozole therapy, variables that could influence results, such as body mass index and aromatase polymorphisms, were not taken into account. However, although the serum FSH level is considered a marker of spermatogenesis and Sertoli cell function (Male Infertility Best Practice Policy Committee of the American Urological Association \& Practice Committee of the American Society for Reproductive Medicine, 2006), only $\mathrm{T}$ levels had a tendency to be correlated with increases in motile sperm count.

Those conclusions must be considered cautiously in light of the small number of patients evaluated. On the other hand, the fact that we are evaluating the same patients before and after treatment, it is an important to increase the reliability of the data obtained.

Another limitation of our study is that we did not measure estradiol and the testosterone/estradiol ( $\mathrm{T} /$ E2) ratio could not be evaluated. However, although letrozole has it main effect when T/E2 is higher than 10, it's action is also observed at lower T/E2 ratios.
Side effects could not be evaluated due to the small sample size. But, three patients reported decreased libido after letrozole therapy. It is also important to point out that PESA is a blind procedure where it is impossible to be certain that the aspiration was carried out the same way for all patients - which could affect the sperm concentration obtained. However, the same surgeon carried out all the procedures using the conventional technique with epididymis aspiration in all sites (head, body and tail), keeping aspiration standardized. In addition, to avoid aspirations bias, we considered motile sperm count and percentage of motility to evaluate the results, as the motility represent quality of sperm and not only concentration, which is not affected by the aspiration procedure.

In summary, the off-label use of letrozole appears to be a reliable treatment for improving sperm recovery for men with OA undergoing PESA-ICSI cycles. This study depicted observational data from 11 patients only. However, considering the promising outcomes observed here, a controlled clinical trial would be warranted to evaluate these results, considering covariables that could influence the efficacy of letrozole in PESA and its possible side effects.

\section{Acknowledgments}

The authors gratefully acknowledge the contributions of the clinical team and the Andrology and IVF Laboratory staffs of the Huntington - Reproductive Medicine, São Paulo, Brazil in regards of patient care and laboratorial procedures. This study did not receive financial support.

\section{CONFLICT OF INTERESTS}

No conflict of interest have been declared.

\section{Corresponding author:}

Mauro Bibancos

Huntington - Reproductive Medicine

São Paulo, SP - Brazil

E-mail: mbibancos@huntington.com.br

\section{REFERENCES}

Aitken RJ, Krausz C. Oxidative stress, DNA damage and the $Y$ chromosome. Reproduction. 2001; 122: 497-506.

Aitken RJ, Sawyer D. The human spermatozoon--not waving but drowning. Adv Exp Med Biol. 2003; 518: 85-98. 
ANVISA - National Health Surveillance Agency. Como a Anvisa vê o uso off label de medicamentos. Brasilia. 2005. Available at:http://www.anvisa.gov.br/medicamentos/ registro/registro_offlabel.htm.

Brodie AM. Aromatase inhibition and its pharmacologic implications. Biochem Pharmacol. 1985. 34: 3213-9.

Cavallini G, Beretta G; Biagiotti G. Preliminary study of letrozole use for improving spermatogenesis in non-obstructive azoospermia patients with normal serum FSH. Asian J Androl. 2011, 13: 895-7.

Cavallini G, Biagiotti G, Bolzon E. Multivariate analysis to predict letrozole efficacy in improving sperm count of non-obstructive azoospermic and cryptozoospermic patients: a pilot study. Asian J Androl. 2013; 15: 806-11.

Dohle GR, Ramos L, Pieters MH, Braat DD, Weber RF. Surgical sperm retrieval and intracytoplasmic sperm injection as treatment of obstructive azoospermia. Hum Reprod. 1998; 13 : 620-3.

Donoso $\mathrm{P}$, Tournaye $\mathrm{H}$, Devroey $\mathrm{P}$. Which is the best sperm retrieval technique for non-obstructive azoospermia? A systematic review. Hum Reprod Update. 2007; 13: 53949.

Foresta C, Selice R, Ferlin A, Garolla A. Recombinant FSH in the treatment of oligozoospermia. Expert Opin Biol Ther. 2009; 9: 659-66.

Garcia-Quevedo L, Blanco J, Sarrate Z, Vidal F. Apoptosis mediated by phosphatidylserine externalization in the elimination of aneuploid germ cells during human spermatogenesis. Andrology. 2014; 2:892-8.

Gregoriou O, Bakas P, Grigoriadis C, Creatsa M, Hassiakos $D$, Creatsas G. Changes in hormonal profile and seminal parameters with use of aromatase inhibitors in management of infertile men with low testosterone to estradiol ratios. Fertil Steril. 2012; 98: 48-51.

Hammoud AO, Griffin J, Meikle AW, Gibson M, Peterson CM, Carrell DT. Association of aromatase (TTTAn) repeat polymorphism length and the relationship between obesity and decreased sperm concentration. Hum Reprod. 2010; 25: 3146-51.

Hsieh MH, Meng MV, Turek PJ. Markov modeling of vasectomy reversal and ART for infertility: how do obstructive interval and female partner age influence cost effectiveness? Fertil Steril. 2007; 88: 840-6.

Kovac JR, Lehmann KJ, Fischer MA. A single-center study examining the outcomes of percutaneous epididymal sperm aspiration in the treatment of obstructive azoospermia. Urol Ann. 2014; 6: 41-5.

Loves S, Ruinemans-Koerts J, de Boer $\mathrm{H}$. Letrozole once a week normalizes serum testosterone in obesity-related male hypogonadism. Eur J Endocrinol. 2008; 158:741-7.

Male Infertility Best Practice Policy Committee of the American Urological Association, Practice Committee of the Amer- ican Society for Reproductive Medicine. Report on evaluation of the azoospermic male. Fertil Steril. 2006; 86: S210-5. Meng MV, Greene KL, Turek PJ. Surgery or assisted reproduction? A decision analysis of treatment costs in male infertility. J Urol. 2005; 174(5): 1926-31.

Patry G, Jarvi K, Grober ED, Lo KC. Use of the aromatase inhibitor letrozole to treat male infertility. Fertil Steril. 2009; 92: 829 e1-2.

Pavlovich CP, King P, Goldstein M, Schlegel PN. Evidence of a treatable endocrinopathy in infertile men. J Urol. 2001; 165: 837-41.

Peng B, Mao Y, Tang XF, Shang Y, Shen CY, Guo Y, Xiang Y, Yang ZW. Comparison of spermatogenic damage induced at 6 months after ligation of the vas deferens at proximal and distal locations in the rabbit. Andrologia. 2011; 43: 129-38.

Raman JD, Schlegel PN. Aromatase inhibitors for male infertility. J Urol. 2002; 167: 624-9.

Rodriguez I, Ody C, Araki K, Garcia I, Vassalli P. An early and massive wave of germinal cell apoptosis is required for the development of functional spermatogenesis. EMBO $\mathrm{J}$. 1997; 16: 2262-70.

Santen RJ. Feedback control of luteinizing hormone and follicle-stimulating hormone secretion by testosterone and estradiol in men: physiological and clinical implications. Clin Biochem. 1981; 14: 243-51.

Saylam B, Efesoy O, Cayan S. The effect of aromatase inhibitor letrozole on body mass index, serum hormones, and sperm parameters in infertile men. Fertil Steril. 2011; 95: 809-11.

Schlegel PN. Aromatase inhibitors for male infertility. Fertil Steril. 2012; 98: 1359-62.

Selice R, Garolla A, Pengo M, Caretta N, Ferlin A, Foresta C. The response to FSH treatment in oligozoospermic men depends on FSH receptor gene polymorphisms. Int J Androl. 2011; 34: 306-12.

Shiraishi K, Takihara H, Naito K. Quantitative analysis of testicular interstitial fibrosis after vasectomy in humans. Aktuelle Urol. 2003; 34: 262-4.

Sunderam S, Kissin DM, Crawford S, Anderson JE, Folger SG, Jamieson DJ, Barfield W D, Division of Reproductive Health, National Center for Chronic Disease Prevention and Health Promotion, CDC. Assisted reproductive technology surveillance - United States, 2010. MMWR Surveill Summ. 2013; 62: 1-24.

Van Perperstraten AM, Proctor ML, Phillipson G, Johnson NP. Techniques for surgical retrieval of sperm prior to ICSI for azoospermia. Cochrane Database Syst Rev. 2001: CD002807.

Zhao D, Pan L, Zhang F, Pan F, Ma J, Zhang X, Liu Y. Successful use of aromatase inhibitor letrozole in NOA with an elevated FSH level: a case report. Andrologia. 2014; 46: 456-7. 